\title{
Mejoramiento de la línea de producción en la fabricación de pallets mediante el estudio de trabajo en Tropical Pallets S.A.
}

\author{
Improvement of pallet manufacturing production-line through work study in the Tropical Pallets company
}

Taipe Chingo Luis ${ }^{1}$ (iD

Universidad Técnica Estatal de Quevedo

Rivas Sierra Danny ${ }^{2}$ (D)

Universidad Técnica Estatal de Quevedo

Fecha recepción: 15 de junio de 2020

Fecha aceptación: 16 de julio de 2020

\section{RESUMEN}

El presente proyecto de investigación se realizó en la empresa Tropical Pallets, con el objetivo de mejorar la línea de producción en la fabricación de los pallets mediante el estudio de trabajo; para esto, se realizó un estudio de todos los elementos que intervienen en el área

\begin{abstract}
This research project was carried out at the Tropical Pallets company, with the aim of improving the production line in the manufacture of pallets through the work study; For this, a study of all the elements that intervene in the production area was carried out, such as: machines and
\end{abstract}

\footnotetext{
1 Ingeniero Industrial, Universidad Técnica Estatal de Quevedo, Facultad de Ciencias de Ingeniería, Quevedo, Ecuador, Iuis.taipe2013@uteq.edu.ec.

2 Máster en Ingeniería de Producción, Universidad Técnica Estatal de Quevedo, Facultad de Ciencias de Ingeniería, Quevedo, Ecuador, drivas@uteq.edu.ec, https://orcid.org/0000-0003-1262-5115
} 
producción como: las máquinas y equipos, los operarios y puestos de trabajo, que en relación de todos estos elementos pueden provocar retrasos en la producción y posibles accidentes laborales. La realización de un estudio de trabajo permite determinar de qué manera se realizan los trabajos en cada proceso, además ayuda a determinar tiempos estándar, para obtener resultados favorables a diferencia del tiempo normal, eliminando tiempos perdidos y posibles cuello de botella. Mediante la observación directa al área de producción se pudo constatar: la existencia de una mala distribución de equipos y maquinarias a distancias innecesarias que incide en el tiempo dedicado a la producción; la mala colocación de los residuos y productos terminados que dificulta la fácil movilidad entre las diferentes etapas de producción. Para el desarrollo de esta problemática se hace uso de herramientas de estudio como; diagramas de flujo, diagrama de recorrido, diagrama analítico y gráficos que permiten determinar la situación actual de la empresa. Este estudio y la redistribución de la planta permitió disminuir considerablemente el tiempo de proceso de los pallets, de 21 minutos a 13,5 minutos por unidad, es decir un ahorro del $36 \%$ de tiempo, con la consecuente reducción en costos e incremento en las ganancias.

PALABRAS CLAVE: Estudio de trabajo, tiempo estándar, Layout, pallets

\section{INTRODUCCIÓN}

El propósito general de este trabajo de investigación es mejorar el proceso de la línea equipment, operators and jobs, which in relation to all these elements can cause delays in production and possible work accidents. Carrying out a work study makes it possible to determine how the work is carried out in each process, it also helps to determine standard times, to obtain favorable results in contrast to the normal time, eliminating lost times and possible bottlenecks. Through direct observation of the production area, it was possible to verify: the existence of a poor distribution of equipment and machinery at unnecessary distances that affects the time dedicated to production; poor placement of waste and finished products that hinders easy mobility between the different stages of production. For the development of this problem, study tools such as; flow charts, path diagram, analytical diagram and charts that allow to determine the current situation of the company. This study and the redistribution of the plant allowed to considerably reduce the processing time of the pallets, from 21 minutes to 13.5 minutes per unit, that is to say, a saving of $36 \%$ of time, with the consequent reduction in costs and increase in the Profits.

KEYWORDS: Work study, standard time, Layout, pallet.

de producción en la fabricación de pallets mediante el estudio de trabajo dentro de los procesos en la empresa Tropical Pallets del cantón Quevedo, se busca determinar tiempos 
reales que permitan establecer una planificación y control en el proceso de fabricación de su producto, eliminando tiempos perdidos y posibles cuellos de botella. Se realiza el análisis de la situación actual de la empresa considerando el proceso de fabricación de los pallets, mismo que permite determinar el modo actual de trabajo y el ritmo de desempeño laboral. De esta manera se busca mejorar la línea de producción con la aplicación de estudios de tiempo por cada proceso, permitiendo la determinación de los tiempos estándar para su respectivo análisis y enfocarnos en la solución a los problemas encontrados, con la finalidad de crear una línea de producción más eficiente y cumplir con la demanda en menor tiempo.

Mediante la observación directa al área de producción de la empresa se ha evidenciado posibles inconvenientes al momento de la fabricación de los pallets como: la falta de un control constante en la producción provocando demoras en su proceso; condiciones ergonómicas inapropiadas para los trabajadores, aumentando la fatiga por agotamiento físico, misma que disminuye la eficiencia de la mano de obra; también la presencia de materia prima defectuosa provocando retraso y paro en la producción. Además, la mala distribución de equipos y maquinarias con relación al área donde se lleva a cabo el proceso de fabricación; tomando en cuenta también la mala colocación de los residuos, provocando el aumento de tiempo de transporte del material desde una máquina a otra. Todos estos problemas mencionados entre otros, ocasionan el incumplimiento de entrega de piezas dentro del plazo requerido en cada área de producción, provocando pérdidas para la empresa.

El estudio de trabajo que se aplica a las distintas fases de producción permite alcanzar resultados inmediatos en la producción, además, se utiliza diversas metodologías y técnicas de investigación que ayudan a la recopilación de datos y la obtención de información necesaria para un diagnostico actual de la empresa, como: diagrama de corrido del material y diagrama flujo de proceso que permite la tabulación de datos de proceso, para el posterior planteamiento de una propuesta de mejora a la línea de producción.

\section{MATERIALES Y MÉTODOS.}

Mediante la observación directa a la empresa en el área de producción, se pudo conocer el método de trabajo actual, para ello se hace uso de una cámara fotográfica, se realiza un dialogo directo con el gerente de la empresa con preguntas relacionados al tema de investigación, el uso de fichas de estudio ayudó a la tabulación de datos e información obtenida 
en la empresa a través de tablas de toma de tiempo, cursograma, diagrama de recorrido y otros gráficos. En el análisis de estos datos obtenidos en el proceso de fabricación de los pallets se determina que la empresa actualmente fabrica 250 pallets a un ritmo de trabajo de 8 horas por día y por último se hace uso del programa Excel para la tabulación de los tiempos obtenidos de las diferentes etapas, mismos que mediante la introducción de algunas fórmulas se obtienen tiempo total, tiempo promedio, y el tiempo estándar.

Según el sistema de calificación Westinghouse, método utilizado por la mayor parte de los analistas en los estudios de tiempos se utilizan cuatro factores para calificar al operario las cuales son: Habilidad, Esfuerzo, Condiciones y Consistencia (Jananía Abraham, 2008). La asignación de suplementos y holguras, se la realiza mediante un análisis de dos causas: asignables por retrasos personales y por fatiga (NIEBEL \& Freivalds, 2009)

\section{RESULTADOS}

A través del diagrama Ishikawa causaefecto se puede examinar ciertos inconvenientes al momento de su producción que estas provocan demoras en el proceso de producción ya sea por el método de trabajo, por la mano de obra, los materiales, la medición de trabajo, el ambiente de trabajo y sus máquinas.

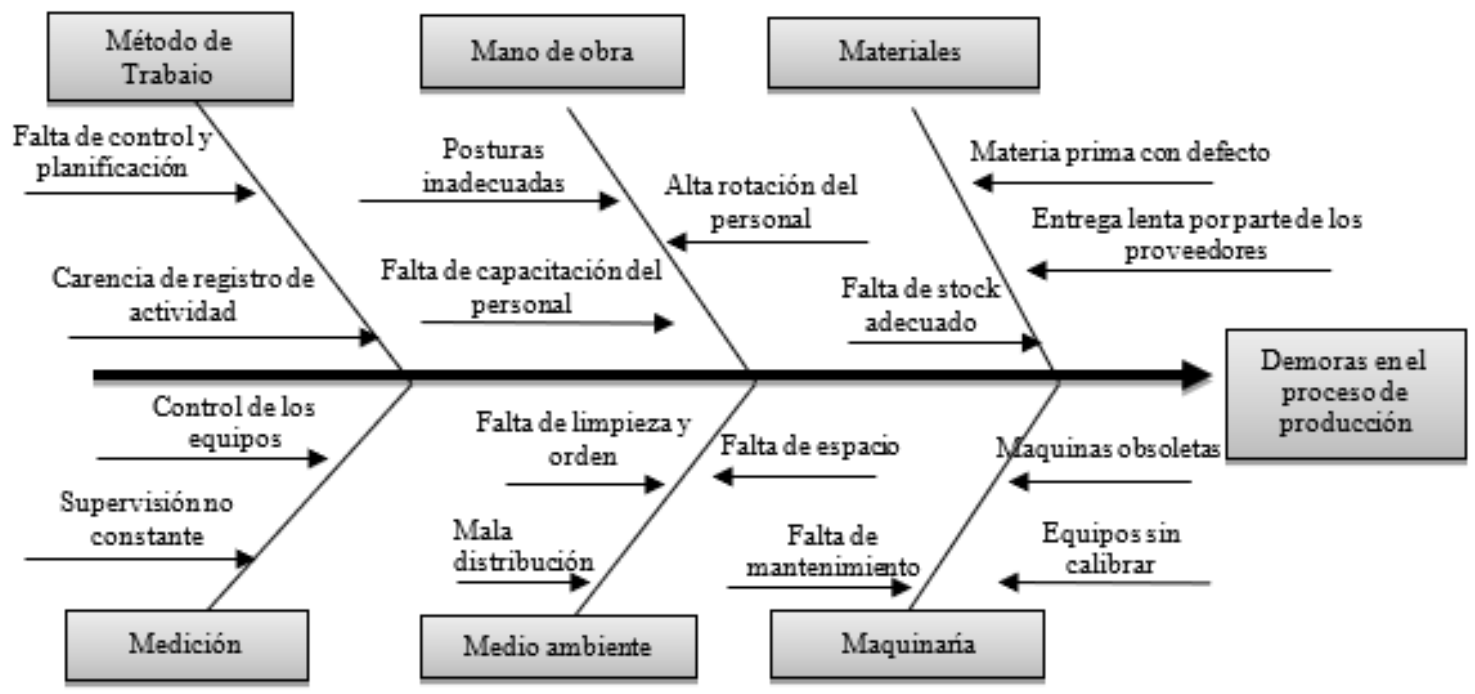


Figura 1: Diagrama causa y efecto de la producción

Figure 1: Cause and effect Diagram

Fuente: elaboración propia

Los materiales de la Tabla 1 son fabricados en la misma empresa, los tamaños de los pallets varían dependiendo el diseño que se va a fabricar:

Tabla 1: Materiales para la fabricación de los pallets

\begin{tabular}{|c|c|c|}
\hline Elementos & Gráfico & Descripción \\
\hline Tablas & & $\begin{array}{l}(122 \mathrm{~cm}, 102 \mathrm{~cm}, 130 \mathrm{~cm} \text { y } 160 \mathrm{~cm}) \text { de largo } \times 10 \mathrm{~cm} \text { de ancho } \\
1 \text { a } 2 \text { pulgada }\end{array}$ \\
\hline Tablillas & & $\begin{array}{l}(122 \mathrm{~cm}, 102 \mathrm{~cm}, 130 \mathrm{~cm} \text { y } 160 \mathrm{~cm}) \text { de largo } x 5 \mathrm{~cm} \text { de ancho } \\
1 \text { a } 2 \text { pulgadas }\end{array}$ \\
\hline Tucos & & 3.3 pulgadas $\mathrm{x} 4$ lados \\
\hline Clavos & & $2^{1 / 2}$ pulgada \\
\hline
\end{tabular}

El proceso de producción de los palletes inicia con la recepción de los materiales, donde se verifican y controlan las trozan de madera que llegan a la empresa que van desde 500 a 1000 unidades, segundo pasan al proceso de aserrado donde las trozan con colocadas en una cierra circular eléctrica para separar la corteza dejando una material cuadrado de acuerdo al diseño a fabricar, después llega al proceso de tableado donde se cortan en tiras de tablas, tablillas y tucos que van de 1 a 4 pulgadas con distintos tamaños de longitud, siguiendo con el proceso, llegan al área de preparación donde cada elemento es cepillado, cortado a medidas específicas de acuerdo al modelo a fabricar; todos estos elementos llegan al proceso de ensamblaje donde se forman los pallets utilizando clavos que son disparados por una enclavadora a presión de 40 a 70 psi, los últimos pasos consisten en almacenar los pallets para proceder con el fumigado, el proceso de secado en la cámara térmica y seguido al área de exhibición y venta. 


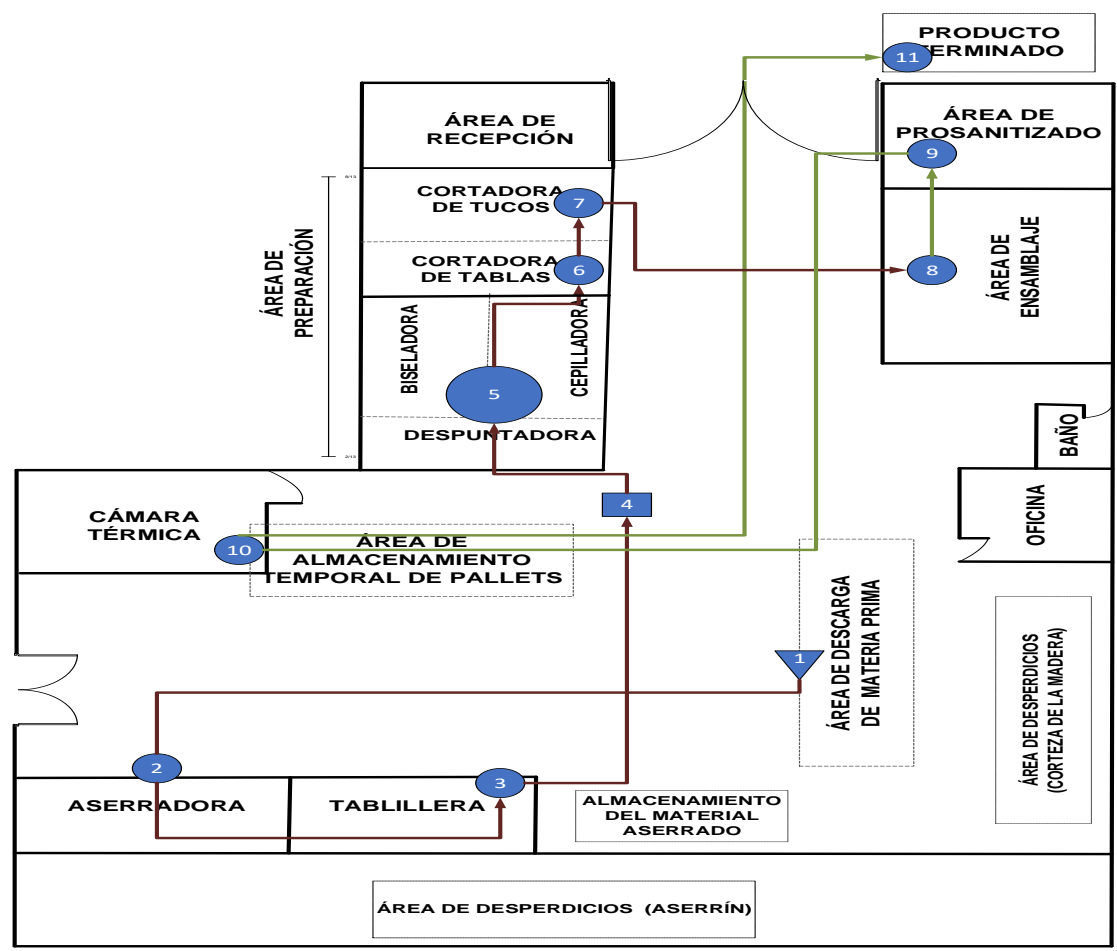

Figura 2: Diagrama de Recorrido

Figure 2: Routing Diagram

Fuente: elaboración propia

El diagrama de recorrido del material que se muestra en el Gráfico 2, indica que existe tráficos cruzados en la producción, por lo cual se sugiere realizar una propuesta de distribución en planta que ayude a la eliminación de dichos tráficos cruzados a fin de mejorar la producción con menos tiempo en el proceso y la reducción de distancias entre los procesos.

Tabla 2: Cursograma analítico del proceso de fabricación de los pallets

\begin{tabular}{|c|c|}
\hline \multicolumn{2}{|c|}{ DIAGRAMA DE ACTIVIDADES } \\
\hline Diagrama № 1 Hoja: 1 de 2 & \\
\hline $\begin{array}{l}\text { Ubicación: } \\
\text { Tropical Pallets S.A. }\end{array}$ & Resumen \\
\hline
\end{tabular}




\begin{tabular}{|c|c|c|c|c|c|c|}
\hline \multicolumn{2}{|l|}{$\begin{array}{l}\text { Actividad: } \\
\text { Fabricación de Pallets }\end{array}$} & \multicolumn{2}{|c|}{ Elemento } & Presente & Propuesto & Ahorros \\
\hline \multicolumn{2}{|l|}{ Fecha: enero/2020 } & \multicolumn{2}{|c|}{ Operación } & 10 & & \\
\hline Operador: 14 & Analista: Taipe Fredy & \multicolumn{2}{|c|}{ Transporte } & 7 & & \\
\hline \multicolumn{2}{|l|}{ Lugar: Área de producción } & \multicolumn{2}{|l|}{ Retrasos } & & & \\
\hline \multicolumn{2}{|l|}{ Método: } & \multicolumn{2}{|c|}{ Inspección } & 1 & & \\
\hline \multicolumn{2}{|l|}{ Presente/Propuesto } & \multicolumn{2}{|c|}{ Almacenamiento } & 2 & & \\
\hline \multicolumn{2}{|l|}{ Tipo: Trabajador/Material/Máquina } & \multicolumn{2}{|c|}{ Tiempo (min) } & 198,01 & & \\
\hline \multirow{2}{*}{\multicolumn{2}{|c|}{$\begin{array}{l}\text { Aprobado por: } \\
\text { Sr. Carlos Murillo }\end{array}$}} & \multicolumn{2}{|c|}{ Distancia (m) } & 161 & & \\
\hline & & \multicolumn{2}{|l|}{ Costo } & & & \\
\hline Descripción de los elementos & \multicolumn{2}{|c|}{ Símbolo } & $\begin{array}{l}\text { Tiempo } \\
\text { (min) }\end{array}$ & $\begin{array}{c}\text { Distancia } \\
\text { (m) }\end{array}$ & \multicolumn{2}{|c|}{ Observación } \\
\hline Selección del material & $\infty$ & $\square \nabla$ & 0,2 & & & \\
\hline $\begin{array}{l}\text { Trasporte de las trozas al área de } \\
\text { aserrío }\end{array}$ & & & 0,65 & 16,6 & \multicolumn{2}{|c|}{$\begin{array}{l}\text { Se lo realiza en forma } \\
\text { manual }\end{array}$} \\
\hline $\begin{array}{l}\text { Aserramiento de trozas grades de } \\
30 \mathrm{~cm} \text { a } 43 \mathrm{~cm} \text { de diámetro }\end{array}$ & & $\nabla$ & 1,53 & & \multicolumn{2}{|c|}{$\begin{array}{l}\text { lo realizan con } 3 \\
\text { operadores }\end{array}$} \\
\hline $\begin{array}{l}\text { Aserramiento de trozas pequeñas } \\
\text { de } 12 \mathrm{~cm} \text { a } 29 \mathrm{~cm} \text { de diámetro }\end{array}$ & $\Phi$ & $\square \vee$ & 1,34 & & \multicolumn{2}{|c|}{$\begin{array}{l}\text { Para esto solo trabajan } 2 \\
\text { operadores }\end{array}$} \\
\hline Trasporte de la madera aserrada & & $\square \nabla$ & 0,32 & 4 & \multicolumn{2}{|c|}{$\begin{array}{l}\text { De forma manual un solo } \\
\text { operador }\end{array}$} \\
\hline $\begin{array}{l}\text { Corte de trozas en tablas, tablillas } \\
\text { y/o tucos sin dimensión especifica }\end{array}$ & $\square$ & $\square \nabla$ & 0.26 & & & \\
\hline Trasponte al área de preparación & & & 3,07 & 28,8 & \multicolumn{2}{|c|}{ Elevador monta carga } \\
\hline Inspección & & $\square \nabla$ & 0,18 & & & \\
\hline Cepillado de tucos & & & 0,57 & & $\begin{array}{l}\text { Más de } 2 \text { pas } \\
\text { maquina }\end{array}$ & las por la \\
\hline Cepillado de tabla y tablillas & & $\square \vee$ & 0.50 & & $\begin{array}{l}\text { Una sola pas } \\
\text { maquina }\end{array}$ & a por la \\
\hline Transporte al área de corte & $\diamond$ & $\square \vee$ & 1,31 & 9,3 & Elevador mo & a carga \\
\hline $\begin{array}{l}\text { Corte de tablillas con } \\
\text { dimensiones de } 102 \mathrm{~cm} \text { y } 122 \mathrm{~cm}\end{array}$ & $0_{1}^{1}$ & $\square \nabla$ & 0.54 & & En fila de 10 & idades \\
\hline $\begin{array}{l}\text { Corte de tucos con dimensiones } \\
\text { de } 4.70 \mathrm{~cm}\end{array}$ & br & $\square \nabla$ & 0,21 & & Una por una & \\
\hline Trasporte al área de ensamblaje & 0 & $\square \nabla$ & 1,24 & 5,4 & Elevador mo & a carga \\
\hline
\end{tabular}




\begin{tabular}{|l|c|c|c|c|}
\hline Construcción de los pallets & 2 operarios cada uno con \\
Trasporte al área de satinado o \\
fumigado
\end{tabular}

Fuente: elaboración propia

Mediante del diagrama analítico de la Tabla 2, se identifica el número de operaciones que se realiza durante todo el proceso de fabricación, también el tiempo que se demoran en cada etapa y la distancia que existe entre las mismas. Con la determinación de los tiempos estándar de cada proceso en la tabla 3 y el diagrama de recorrido actual de producción, se identificó que existen retrasos o cuellos de botella en las siguientes etapas: transporte de las trozas de madera que van desde el área de recepción de la materia prima hacia el área de aserrado; transporte del material que van desde el área de tablillado hacia el área de preparación; transporte de los pallets desde el área de prosanitizado, al área de cámara de secado. 
Tabla 3: Tiempo estándar de las etapas de producción

\begin{tabular}{|c|c|c|c|c|c|c|}
\hline LINEA DE PRODUCCION DE LA FABRICACIÓN DE PALLET & & & & & $\begin{array}{l}\text { fecha: } 29 / 01 \\
\text { Taks- Time: } \\
\text { Número de } \\
14\end{array}$ & $\frac{1020}{1,9 \mathrm{~min}}$ \\
\hline \multirow[t]{2}{*}{ Etapas de proceso } & № observaciones & $\begin{array}{l}\text { tiempo } \\
\text { promedio }\end{array}$ & $\begin{array}{l}\text { factor de } \\
\text { calificación }\end{array}$ & $\begin{array}{l}\text { tiempo } \\
\text { normal }\end{array}$ & suplemento & $\begin{array}{l}\text { tiempo } \\
\text { estándar }\end{array}$ \\
\hline & & minutos & $\%$ & & $\%$ & \\
\hline $\begin{array}{l}\text { Selección del material } \\
\text { Trasporte de las trozas al área de aserrio } \\
\text { Aserramiento de trozas grades de } 30 \mathrm{~cm} \text { a } 43 \mathrm{~cm} \text { de diámetro } \\
\text { Aserramiento de trozas pequeñas de } 12 \mathrm{~cm} \mathrm{a} 29 \mathrm{~cm} \text { de diámetro } \\
\text { Trasporte de la madera aserrada } \\
\text { Corte de trozas en tablas, tablillas y/o tucos sin dimensión especifica } \\
\text { Trasponte al área de preparación } \\
\text { Cepillado de tabla y tablillas } \\
\text { Cepillado de tucos } \\
\text { Transporte al área de corte } \\
\text { Corte de tablillas con dimensiones de } 102 \mathrm{~cm} \text { y } 122 \mathrm{~cm} \\
\text { Corte de tucos con dimensiones de } 4.70 \mathrm{~cm} \\
\text { Trasporte al área de ensamblaje } \\
\text { Construcción de los pallets } \\
\text { Trasporte al área de Pretratamiento } \\
\text { Trasporte al área de Secado en la cámara témica }\end{array}$ & $\begin{array}{l}20 \\
20 \\
20 \\
20 \\
20 \\
20 \\
10 \\
20 \\
20 \\
10 \\
20 \\
20 \\
10 \\
20 \\
10 \\
10\end{array}$ & $\begin{array}{l}0,2025 \\
0,6550 \\
1,5308 \\
1,3450 \\
0,3225 \\
0,2625 \\
3,0767 \\
0,5008 \\
0,5667 \\
1,3067 \\
0,5367 \\
0,2167 \\
1,2433 \\
1,4950 \\
1,3700 \\
3,6383\end{array}$ & $\begin{array}{l}96 \\
96 \\
96 \\
96 \\
96 \\
96 \\
96 \\
96 \\
96 \\
96 \\
96 \\
96 \\
96 \\
96 \\
96 \\
96\end{array}$ & $\begin{array}{l}0,1944 \\
0,6288 \\
1,4696 \\
1,2912 \\
0,3096 \\
0,2520 \\
2,9536 \\
0,4808 \\
0,5440 \\
1,2544 \\
0,5152 \\
0,2080 \\
1,1936 \\
1,4352 \\
1,3152 \\
3,4928\end{array}$ & $\begin{array}{l}20 \\
20 \\
20 \\
20 \\
20 \\
20 \\
20 \\
20 \\
20 \\
20 \\
20 \\
20 \\
20 \\
18 \\
20 \\
20\end{array}$ & $\begin{array}{l}0,23 \\
0,75 \\
1,76 \\
1,55 \\
0,37 \\
0,30 \\
3,54 \\
0,58 \\
0,65 \\
1,51 \\
0,62 \\
0,25 \\
1,43 \\
1,69 \\
1,58 \\
4,19\end{array}$ \\
\hline Trasporte al área de Secado en la cámara témica & & \multicolumn{5}{|c|}{ TOMA DE TIEMPO POR HORA } \\
\hline $\begin{array}{l}\text { Fumigado y curado } \\
\text { Proceso de Tiempo de secado } \\
\text { Almacenamiento de producto terminado }\end{array}$ & $\begin{array}{l}3 \\
0 \\
0\end{array}$ & $\begin{array}{l}0,2306 \\
3 \\
4\end{array}$ & $\begin{array}{l}96 \\
100 \\
100\end{array}$ & $\begin{array}{l}0,2213 \\
3,0000 \\
4,0000\end{array}$ & $\begin{array}{l}20 \\
0 \\
0\end{array}$ & $\begin{array}{l}0,27 \mathrm{~h} \\
3 \mathrm{~h} \\
4 \mathrm{~h}\end{array}$ \\
\hline
\end{tabular}

Para lo cual se realiza una propuesta de redistribución en planta para lograr la disminución de tiempo de producción, flexibilidad en el proceso, cumpliendo con la demanda en menor tiempo con la misma calidad. De la misma manera se busca disminuir los tráficos cruzados entre los procesos y la distancia que existe entre ellos. En el proceso de curado o fumigado, la cámara de secado y la exhibición del producto terminado el tiempo se mantiene debido a que estos últimos pasos se realizan una vez completado el número de productos requeridos. A continuación, se presenta la propuesta de un nuevo modelo de línea de producción que optimice los tiempos en la fabricación de los pallets. 


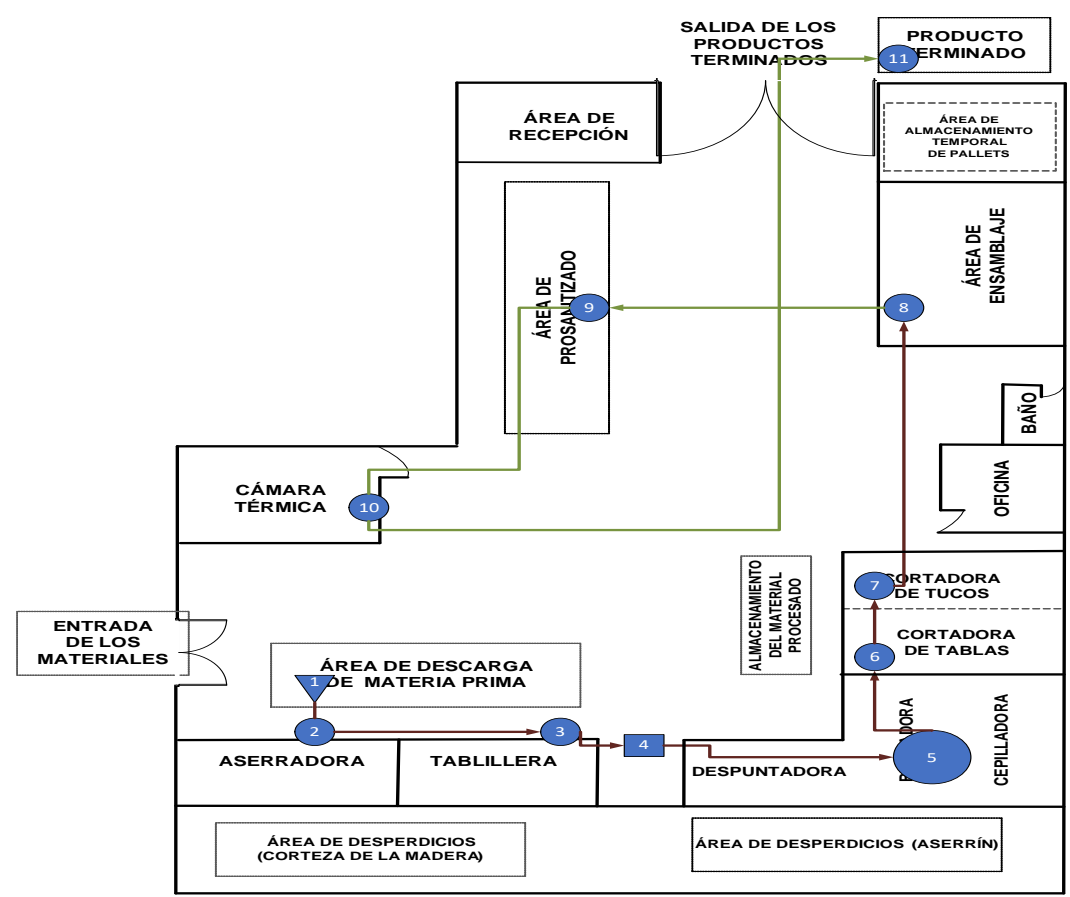

Figura 3: Diagrama de Recorrido Propuesto

Figure 2: Proposed Routing Diagram

Fuente: elaboración propia

En la Figura 3 se puede identificar que existe mejoras en la distribución debido a que se elimina el proceso de transporte de la materia prima hacia el área de aserrío, existen menos número de tráfico cruzados y la distancia entre el proceso de Aserrío con el proceso de Preparación disminuye, también está el área de Prosanitizado con el cuarto de Cámara Térmica. Con la nueva distribución se logrará evitar retrasos en la línea de producción, debido a que se mejora el ritmo de producción teniendo como factor de calificación trabajo de
$105 \%$ a diferencia del actual que está en el $96 \%$, se puede analizar y establecer un nuevo porcentaje en los suplementos o tolerancia que se aplican al trabajador porque se reduce el proceso de transporte del material del área de aserrío al área de preparación, que esto significaría que el trabajador tendría una holgura por posición normal del 0\% que es una posición un poco incomoda según las Holguras recomendadas por ILO (NIEBEL \& Freivalds, 2009). 
Tabla 4: Diagrama analítico propuesto (Resumen)

\begin{tabular}{|l|l|l|l|}
\hline \multicolumn{3}{|c|}{ Resumen } \\
\hline \multicolumn{1}{|c|}{ Elemento } & Presente & Propuesto & Ahorros \\
\hline Operación & 10 & 10 & 0 \\
\hline Transporte & 7 & 6 & 1 \\
\hline Retrasos & & & \\
\hline Inspección & 1 & 1 & 0 \\
\hline Almacenamiento & 2 & 2 & 0 \\
\hline Tiempo (min) & & & \\
\hline Distancia (m) & 161 & 82,1 & 79,9 \\
\hline
\end{tabular}

Fuente: elaboración propia

A continuación, se determinará el tiempo estándar para la propuesta de distribución:

Tabla 5: Tiempo estándar para la distribución propuesta

\begin{tabular}{|c|c|c|c|c|c|c|}
\hline \multicolumn{5}{|c|}{ LINEA DE PRODUCCION EN LA FABRICACION DE PALLETS } & \multicolumn{2}{|c|}{$\begin{array}{l}\text { Fecha: } \mathbf{2 9 / 0 1 / 2 0 2 0} \\
\text { Tack time: } 1,9 \text { minutos } \\
\text { Número de operador: } 14\end{array}$} \\
\hline Etapas de proceso de fabricación & $\begin{array}{c}\text { № } \\
\text { Observaciones }\end{array}$ & $\begin{array}{l}\text { Tiempo } \\
\text { promedio } \\
\text { Minutos }\end{array}$ & $\begin{array}{l}\text { Factor de } \\
\text { calificación } \\
\%\end{array}$ & $\begin{array}{l}\text { Tiempo } \\
\text { normal }\end{array}$ & $\begin{array}{c}\text { Suplemento } \\
\%\end{array}$ & $\begin{array}{l}\text { Tiempo } \\
\text { estándar }\end{array}$ \\
\hline Selección del material & 20 & 0,2025 & 105 & 0,2126 & 16 & 0,25 \\
\hline Aserramiento de trozas grades de $30 \mathrm{~cm}$ a $43 \mathrm{~cm}$ de diámetro & 20 & 1,5308 & 105 & 1,6074 & 16 & 1,86 \\
\hline Aserramiento de trozas pequeñas de $12 \mathrm{~cm}$ a $29 \mathrm{~cm}$ de diámetro & 20 & 1,3450 & 105 & 1,4123 & 16 & 1,64 \\
\hline Trasporte de la madera aserrada & 20 & 0,3225 & 105 & 0,3386 & 16 & 0,39 \\
\hline Corte de trozas en tablas, tablillas $\mathrm{y} / \mathrm{o}$ tucos sin dimensión especifica & 20 & 0,2600 & 105 & 0,2730 & 16 & 0,32 \\
\hline Trasponte al área de preparación & 10 & 0,3200 & 105 & 0,3360 & 16 & 0,39 \\
\hline Cepillado de tabla y tablillas & 20 & 0,5008 & 105 & 0,5259 & 16 & 0,61 \\
\hline Cepillado de tucos & 20 & 0,5667 & 105 & 0,5950 & 16 & 0,69 \\
\hline Transporte al área de corte & 10 & 0,2100 & 105 & 0,2205 & 16 & 0,26 \\
\hline Corte de tablillas con dimensiones de $102 \mathrm{~cm}$ y $122 \mathrm{~cm}$ & 20 & 0,5367 & 105 & 0,5635 & 16 & 0,65 \\
\hline Corte de tucos con dimensiones de $4.70 \mathrm{~cm}$ & 20 & 0,2167 & 105 & 0,2275 & 16 & 0,26 \\
\hline Trasporte al área de ensamblaje & 10 & 1,6100 & 105 & 1,6905 & 16 & 1,96 \\
\hline Construcción de los pallets & 20 & 1,4950 & 105 & 1,5698 & 18 & 1,85 \\
\hline & 10 & 1,2400 & 105 & 1,3020 & 16 & 1,51 \\
\hline \multirow{2}{*}{ Trasporte al área de Secado en la cámara térmica } & 10 & 0,5400 & 105 & 0,5670 & 16 & 0,66 \\
\hline & & \multicolumn{5}{|c|}{ TOMA DE TIEMPO POR HORA } \\
\hline Fumigado y curado & 3 & 0,2306 & 96 & 0,2213 & 16 & $0,27 \mathrm{~h}$ \\
\hline Proceso de Tiempo de secado & 0 & 3 & 100 & 3,0000 & 0 & 3,00 \\
\hline Almacenamiento de producto terminado & 0 & 4 & 100 & 4,0000 & 0 & 4,00 \\
\hline
\end{tabular}


Fuente: elaboración propia

Ahora se comparará la línea de producción actual vs la propuesta:

Tabla 6: Tabla comparativa

\begin{tabular}{|c|c|c|}
\hline \multirow{2}{*}{ ETAPAS DE FABRICACIÓN DE LOS PALLETS } & \multicolumn{2}{|c|}{ Tiempo estándar } \\
\hline & Presente & Propuesto \\
\hline Selección del material & 0,23 & 0.25 \\
\hline Trasporte de las trozas al área de aserrío & 0,75 & \\
\hline Aserramiento de trozas grades de $30 \mathrm{~cm}$ a $43 \mathrm{~cm}$ de diámetro & 1,76 & 1,86 \\
\hline Aserramiento de trozas pequeñas de $12 \mathrm{~cm}$ a $29 \mathrm{~cm}$ de diámetro & 1,55 & 1,64 \\
\hline Trasporte de la madera aserrada & 0,37 & 0,39 \\
\hline Corte de trozas en tablas, tablillas y/o tucos sin dimensión especifica & 0,3 & 0,32 \\
\hline Trasponte al área de preparación & 3,54 & 0,39 \\
\hline Cepillado de tabla y tablillas & 0,58 & 0,61 \\
\hline Cepillado de tucos & 0,65 & 0,69 \\
\hline Transporte al área de corte & 1,51 & 0,26 \\
\hline Corte de tablillas con dimensiones de $102 \mathrm{~cm}$ y $122 \mathrm{~cm}$ & 0,62 & 0,65 \\
\hline Corte de tucos con dimensiones de $4.70 \mathrm{~cm}$ & 0,25 & 0,26 \\
\hline Trasporte al área de ensamblaje & 1,43 & 1,96 \\
\hline Construcción de los pallets & 1,69 & 1,85 \\
\hline Trasporte al área de Pretratamiento & 1,58 & 1,51 \\
\hline Trasporte al área de Secado en la cámara térmica & 4,19 & 0,66 \\
\hline TOTAL & 21 & 13,05 \\
\hline
\end{tabular}

Fuente: elaboración propia 
Con la aplicación de tiempo estándar en las etapas de trabajo actual a diferencia de las etapas de producción propuesto podemos determinar que existe una diferencia de 7,95 minutos considerando los datos que se muestra en la Tabla 6, esto nos permitirá fabricar 15 unidades más en 8 horas de trabajo, por otra parte, se podrá cumplir con el pedido en menos tiempo.

\section{DISCUSIÓN}

Según Kanawaty, G en su libro Introducción al Estudio de Trabajo (Kanawaty, 1996), la medición del trabajo es la aplicación de técnicas para determinar el tiempo que interviene un trabajador calificado en llevar a cabo una tarea según una norma de rendimiento preestablecida. Considerando lo anterior, se identificó el tiempo que demora por proceso, encontrando tiempos excesivos al momento del trasporte de la materia prima realizada por los operarios ya se por una mala distribución de equipos o herramientas en el área de producción o por no tener una línea de producción continua. Con la propuesta realizada de una nueva distribución en planta se puede mejorar los tiempos de producción por proceso, según él autor Meyes, Fred E (MEYES, 2000), del libro estudio de tiempos y movimientos menciona que la administración moderna exige de sus ingenieros y técnicos industriales una vigilancia constante a fin de reducir costos y esfuerzos y mejorar el ritmo de trabajo. Dentro de estos procesos se mejoró el procedo de transporte se mejora las condiciones de trabajo de los operarios evitando la fatiga y obteniendo un ritmo de trabajo más eficiente consiguiendo reducir el tiempo de fabricación de 21 minutos a 13,05 minutos.

\section{CONCLUSIONES}

Una vez establecidos los tiempos estándar en cada una de las etapas de producción, aplicado un factor de calificación del 96\%, de acuerdo al análisis efectuado por las condiciones de puesto de trabajo y con el $20 \%$ de los suplementos permitidos al rendimiento del trabajador, se pudo determinar que existen pérdidas de tiempo y movimientos innecesarios en las siguientes etapas: el movimiento de las trozas de madera desde el área de recepción del material al área de aserrío, también el movimiento que se efectúa desde el área de tablillado al área de preparación y por último el movimiento de los pallets desde el área de prosanitizado al área de cámara de secado.

Con la distribución en planta propuesta, se logra mejorar la línea de producción, logrando obtener un proceso más eficiente y consecutivo 
a su vez se mejora las condiciones de trabajo, debido a que se reducen las distancias que existen entre las diferentes etapas detectadas anteriormente, por ende, se reduce el tiempo que se toma para transportar el material y que esto nos da opción a producir más unidades en los tiempos requeridos o a su vez producir los 250 pallets en menos tiempo como se lo muestra en la Tabla 5 de la sección resultados, es decir un ahorro del $36 \%$ de tiempo, con la consecuente reducción en costos e incremento en las ganancias.

\section{REFERENCIAS}

Libro impreso

[1]. Jananía Abraham, C. (2008). Manual de tiempos y movimientos : lngeniería de métodos. México: LIMUSA. S.A de C. V. GRUPO NORIEGA EDITORES.

[2]. Kanawaty, G. (1996). INTRODUCCION AL ESTUDIO DEL TRABAJO. Ginebra: Oficina Internacional del Trabajo.

[3]. MEYES, F. E. (2000). Estudios de Tiempos y movimientos. México: Pearson Educación.

[4]. NIEBEL, B. W., \& Freivalds, A. (2009). Ingeniería industrial: Métodos, estándares y diseño del trabajo (Vol. Duodécima edición). México, D.F.: McGRAWHILL/INTERAMERICANA EDITORES, S.A. DE C.V 